\title{
Ein Japaner in Marburg
}

\author{
Aus den Erinnerungen - Jiden - des japanischen Bakteriologen \\ Taichi Kitashima (1870-1956)
}

\author{
Ulrike Enke und Aeka Ishihara
} A Japanese in Marburg. Excerpts from the Memories - Jiden - of the Japanese Bacteriologist Taichi Kitashima
$(1870-1956)$

\begin{abstract}
The heart of this article is the transmission of selected chapters from the Japanese bacteriologist Taichi Kitashima's (1870-1956) autobiographical memoirs (in Japanese, Jiden) published in 1955, in which Kitashima reports on his stay in Marburg in a very personal and subjective way. Like other Japanese physicians of his generation, Kitashima spent several years in Germany in order to work with the serum researcher Emil von Behring and continued his education there. The contact came through Kitashima's teacher Shibasaburô Kitasato, who had worked with Behring in Berlin on questions of immunology. The memoir gives insight into Behring's laboratory work and his relation to his "subordinates". The editors investigate to what extent Kitashima's assessment, made from a distance of fifty years, of his stay in Germany as "wasted time" was accurate, given the advantages that arose from having been part of a vibrant European scientific community, including encounters in the Pasteur Institute in Paris, and why he cultivated his contacts with Germany and the Behring family during National Socialism.
\end{abstract}

Keywords: Taichi Kitashima, Emil von Behring, Shibasaburô Kitasato, Jiden, Marburg, Japan, snake venom

Im Mittelpunkt des Beitrags steht die Übertragung ausgewählter Kapitel aus den 1955 niedergelegten autobiographischen Erinnerungen (japanisch: Jiden) des japanischen Bakteriologen Taichi Kitashima (1870-1956), in denen Kitashima stark persönlich gefärbt über seinen Aufenthalt in Marburg berichtet. Wie andere japanische Mediziner seiner Generation verbrachte Kitashima mehrere Jahre in Deutschland, um dort bei dem Serumforscher Emil von Behring zu arbeiten und sich weiterzubilden. Der Kontakt war über Kitashimas Lehrer Shibasaburô Kitasato zustande gekommen, mit dem Behring in Berlin über Fragen der Immunologie geforscht hatte. Im „Kommentar" wird diskutiert, inwieweit die von Kitashima in der zeitlichen Distanz von 50 Jahren getroffene Einschätzung des Deutschlandaufenthalts als "vergeudete Zeit" als zutreffend anzusehen ist, welche Vorteile ihm aus den europäischen Kontakten, zu denen auch Begegnungen im Pariser Pasteur-Institut gehörten, erwuchsen und aus welchen Gründen sie im Nationalsozialismus wiederbelebt wurden.

\section{Historische Hintergründe und Überlieferung}

\section{Die japanische Medizin im späten 19. Jahrhundert}

„Die drei Jahre in Marburg waren für mich faktisch umsonst, ich habe nur Behring geholfen und für ihn gearbeitet." Dieses betrübliche Resümee findet sich in den Erinnerungen des japanischen Bakteriologen und späteren 
Direktors des Kitasato-Instituts in Tokio, Taichi Kitashima (1870-1956), der sich ab November 1897 für drei Jahre zu Fortbildungszwecken bei dem Immunologen Emil (von) Behring (1854-1917) in Deutschland aufhielt. Die Erinnerungen, die im Folgenden in Auszügen vorgestellt werden, geben einen sehr persönlichen Einblick in eine Phase der japanischen $\mathrm{Me}$ dizingeschichte, die von einer verstärkten Hinwendung Japans nach Europa geprägt war.

Ab circa 1870 hatten japanische Ärzte und Absolventen der Universität Tokio begonnen, zum Abschluss ihrer medizinischen Ausbildung für einen längeren Zeitraum nach Deutschland zu reisen, um sich in deutschen Universitätsinstituten und -krankenhäusern speziell in Chirurgie, Augenheilkunde, Innerer Medizin und Bakteriologie weiterzubilden. Diese Strömung wurde erst mit Ausbruch des Ersten Weltkriegs beendet. Doch nicht erst durch die von der Meiji-Regierung (1868-1912) geförderte Öffnung nach Westen kam es zu einer Abwendung von der traditionellen, auf chinesische Ursprünge zurückgehende Kampô-Medizin (auch Kanpô, „chinesische Methode“); schon von Anfang an lassen sich Einflüsse aus dem Ausland, etwa aus China, Portugal, Holland und Deutschland, nachweisen. Das ins Land kommende Wissen wurde den japanischen Verhältnissen angepasst und in den bestehenden Wissenskanon integriert (Käser 2011).

Erste intensivere Kontakte zur europäischen Medizin können seit Mitte des 16. Jahrhunderts nachgewiesen werden, als europäische Kaufleute und christliche Missionare in Japan eintrafen und die sie begleitenden jesuitischen Missionsärzte Krankenstationen errichteten. Obwohl in der EdoZeit (1600-1868) die Kontakte zum Ausland stark eingeschränkt wurden, kam es trotz Abschließungspolitik und Einfuhrverboten westlicher Bücher über holländische Handelsstationen zu einem Einstrom westlichen Wissens, das insbesondere die Anatomie und Chirurgie beeinflusste (Käser 2011; Reißenweber 2005: 692).

Während der Edo-Zeit hatten in Japan lebende Ärzte wie Engelbert Kaempfer (1651-1716) und Philipp Franz Balthasar von Siebold (1796-1866) auch die deutsche Medizin in Japan bekannt gemacht. Auf sie berief man sich, als man sich bei der Hinwendung zur europäischen Medizin zwischen der holländischen, englischen und deutschen Richtung entscheiden musste (Käser 2011: 115). Als Movens für die gezielte Förderung der westlichen Medizin in der Meiji-Zeit können unter anderem die auf chirurgische Fähigkeiten zurückgehenden Heilerfolge bei der Behandlung Verwundeter, aber auch die erfolgversprechenden Präventivmaßnahmen bei Infektionskrankheiten genannt werden (Oberländer 1995: 187 f.).

Es ist bekannt, dass es während der Meiji-Periode (1868-1912) drei zeitlich aufeinanderfolgende Gruppen von Medizinern gab, die in der westlichen Medizin ausgebildet wurden (Chen 2010; Käser 2011: 115 f.). Während 
die erste Gruppe die japanischen Studenten und Ärzte umfasste, die in ihrem Heimatland medizinischen Unterricht von deutschen Militär- oder Marineärzten wie Leopold Müller (1824-1893) und Theodor Hoffmann (1837-1894) erhielten, wurde die nachfolgende Generation in den 1880er und den frühen 1890er Jahren nach Deutschland geschickt. Viele japanische Gastmediziner kamen von der medizinischen Fakultät der Kaiserlichen Universität Tokio, dem damals einzigen Institut, an dem man Medizin nach europäischem Vorbild studieren konnte. Von Seiten der japanischen Regierung wurde das Auslandsstudium nicht nur als Aus- und Weiterbildung betrachtet, die jungen Männer galten darüber hinaus auch „als Botschafter der Regierung“, die in Europa Wissen sammeln und Techniken erlernen und nach ihrer Rückkehr dazu beitragen sollten, einen modernen japanischen Staat nach westlichen Vorbild zu errichten (Burks 1985; Chen 2010: 13; Ishihara 2012b).

$\mathrm{Zu}$ den berühmtesten japanischen Wissenschaftlern der zweiten Gruppe gehört Shibasaburô Kitasato (1853-1931), ${ }^{1}$ der als promovierter Arzt auf Vermittlung seines Mentors Masanori Ogata (1853-1919) zu dem Bakteriologen Robert Koch (1843-1910) nach Berlin kam, um hier seine bakteriologischen Kenntnisse zu vertiefen. Tatsächlich gelang es ihm in Kochs hygienischen Institut, als erster den Erreger des Wundstarrkrampfs in Reinkultur zu züchten (Hubenstorf 2006: $191 \mathrm{f}$.).

\section{Behring und Kitasato}

Kitasato kehrte 1892 nach Japan zurück. Nach dem Vorbild Ogatas sandte er ebenfalls besonders befähigte Schüler zur Weiterbildung nach Deutschland, jedoch nicht nach Berlin zu Koch, sondern unter anderem nach Marburg, wo seit 1895 Emil Behring den Lehrstuhl für Hygiene innehatte. Mit Behring hatte Kitasato seit 1889 in Kochs Labor zusammengearbeitet, wo die beiden Wissenschaftler 1890 die Wirkung der Antitoxine gegen Tetanus und Diphtherie hatten nachweisen können. Die gemeinsame Forschungsarbeit mag zu einer engeren persönlichen Verbindung zwischen den fast Gleichaltrigen geführt haben, entscheidender wird jedoch Kitasatos Wertschätzung der wissenschaftlichen und sich in der praktischen Laborarbeit bewährenden Fähigkeiten seines ehemaligen Kollegen gewesen sein. Behring hatte seit der Veröffentlichung der gemeinsamen Arbeit Ueber das Zustandekommen der Diphtherie-Immunität und der Tetanus-Immunität bei Thieren in der Deutschen Medizinische Wochenschrift (Behring \& Kitasato 1890) ein wirksames Heilmittel gegen die Diphtherie entwickelt, das seit 1894 industriell hergestellt wurde und weltweite Aufmerksamkeit erzielte. Im Gegensatz zu Koch, der mit seinem Tuberkulin gescheitert war, konnte Behring mit dem Heilserum ein aus der Laborarbeit stammendes Produkt vorweisen, das äußerst erfolgreich zur Bekämpfung von Infektionskrank- 
heiten eingesetzt wurde. Die Hoffnung auf Teilhabe an anwendungsbezogenem Wissen und an Techniken, die in praxisrelevante Ergebnisse münden, mag ausschlaggebend für Kitasato gewesen sein, einen seiner besten Schüler in Marburg arbeiten zu lassen. Am 28. September 1897 schickte Kitasato ein Empfehlungsschreiben an Behring, das Kitashima mit der Bitte anpries, seinen langjährigen Assistenten in seinen Laboren aufzunehmen (Enke 2010: 179). Die positive Antwort muss umgehend eingetroffen sein: Bereits am 3. Oktober bestieg Kitashima in Yokohama ein deutsches Schiff und traf am 13. November 1897 in Marburg ein, wo er sich mit einigen Unterbrechungen bis zum März 1901 aufhielt (Abb. 1). ${ }^{2}$

\section{Japanische Mediziner in Deutschland}

Der bevorzugte Ort für das Medizinstudium in Deutschland war nicht nur für die unmittelbaren Nachbarn aus dem Osten Europas (Kreuder-Sonnen 2012: 160), sondern auch für Japaner die preußische Hauptstadt Berlin, wo die ältere Generation, also die in den 1870er und 1880er Jahren zurückgekehrten japanischen Professoren, studiert hatte (Hartmann 2000). ${ }^{3}$

Abb. 1 Portraitkarte Shibasaburô Kitasatos mit Widmung „Herrn Stabsarzt Dr. Behring zur freundlichen Erinnerung", Berlin, 21. März 1892 ○ Behring-Archiv Marburg, Sign. EvB/B 70/1

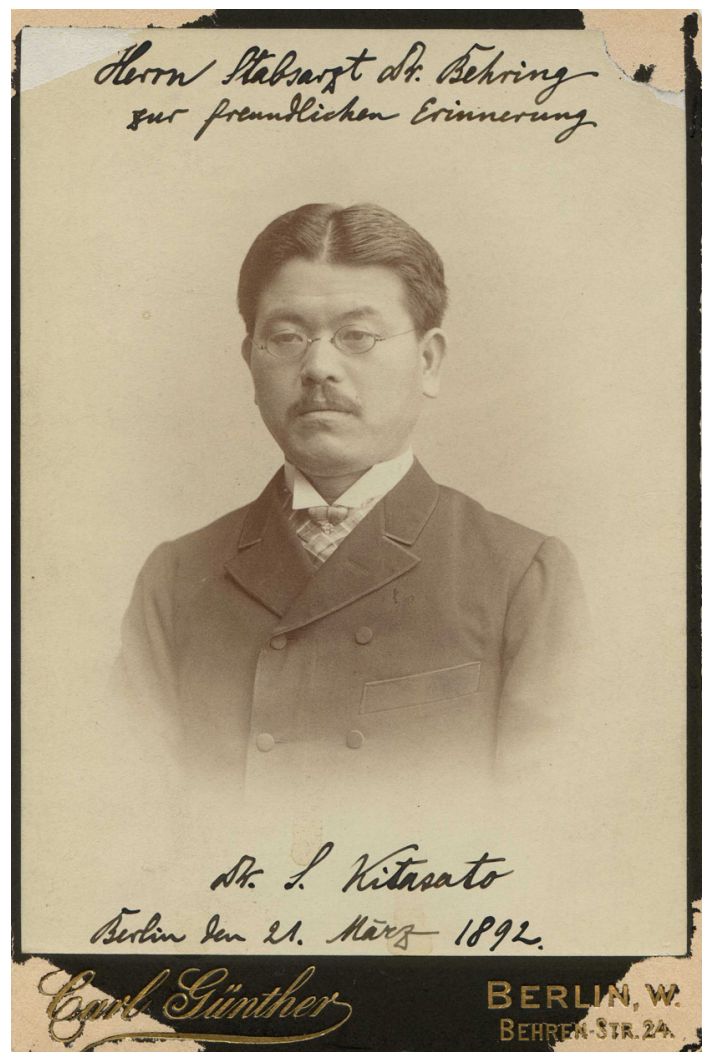


Aber auch Universitätsstädte mit international bekannten Ordinarien waren beliebte Studienorte, die wichtige Orientierungspunkte für die Neuankömmlinge bildeten und funktionierende Netzwerke garantierten (Hartmann 2000; Chen 2010: 89 f.). Zeitgleich mit Kitashima hielten sich in Marburg noch drei weitere japanische Medizinstudenten auf: Jinnosuke Tsuzuki (1869-1933), Hiroshi Watanabe (1865-?) und Sutezô Osaki [Ozaki] (1872-?). ${ }^{4}$ Dank ihrer Deutschkenntnisse, die sie sowohl im Rahmen des in deutscher Sprache durchgeführten Medizinstudiums als auch durch Sprachkurse erworben hatten, waren die japanischen Gäste zumindest theoretisch befähigt, am gesellschaftlichen und akademischen Leben in Deutschland teilzunehmen. Dass ihre aus europäischer Sicht ungewöhnlichen Sitten und Gebräuche - die Begrüßungsrituale und der noch unvertraute Umgang mit Messer und Gabel - durchaus wahrgenommen wurden, zeigen briefliche Äußerungen von Behrings Ehefrau Else von Behring (1876-1936), die nach der ersten Begegnung mit dem fremden Gast notiert: „Eben hat mir d. Japaner Kitashima einen Besuch gemacht. [...] Er begrüßt einen noch ganz japanisch, indem er sich bückt \& mit den Händen seine Beine reibt."

Taichi Kitashima, Jiden

Wie Jane-Hsiu Chen anhand ausgewählter Selbstdokumente japanischer Ärzte gezeigt hat, hinterließen viele japanische Gastwissenschaftler Tagebücher, Reiseberichte und andere autobiographische Erzählungen, die das wissenschaftliche und private Leben in einem fremden Land und einer fremden Kultur schildern (Chen 2010). Über Kitashimas Zeit in Marburg war bisher wenig bekannt, da neben wenigen Briefen (Enke 2010: $182 \mathrm{f}$.) und kurzen Einträgen in das Gästebuch der Familie Behring nur Versuchsprotokolle und drei wissenschaftlichen Publikationen vorliegen, ${ }^{6}$ die einen Einblick in das akademische Umfeld gewähren. Es handelt sich wohl um die einzigen Nachlassdokumente des späteren Direktors des Tokioter Instituts für Infektionskrankheiten, da Kitashima im Laufe seines langen Lebens mehrfach seine Besitztümer, zu denen auch die persönlichen Aufzeichnungen gehörten, durch Erdbeben und Kriegseinwirkungen verlor. Der Verlust dieser Quellen ist bedauerlich, da gerade die Schilderungen und Erinnerungen eines Nicht-Europäers eine neue Perspektive sowohl auf den Forschungsalltag im Umkreis Behrings als auch auf die Bedingungen internationaler Vernetzung hätten bieten können.

Bei Recherchen über Kitasatos Schülerinnen und Schüler, zu denen auch die spätere Augenärztin Tadako Urata (1873-1936) gehört (Ishihara 2012a), stieß die japanische Literaturwissenschaftlerin Aeka Ishihara in der Kitasato-Gedenkstätte in Tokio auf Jiden, die Erinnerungen Kitashimas (Kitashima 1955). Die Schrift ist in Japan bekannt und wird auch in deutschen bio- 
Abb. 2 Taichi Kitashima während der Marburger Zeit; Bildrechte (c) Kitasato-Institut. Abdruck mit freundl. Genehmigung des KitasatoInstituts Tokyo/Japan

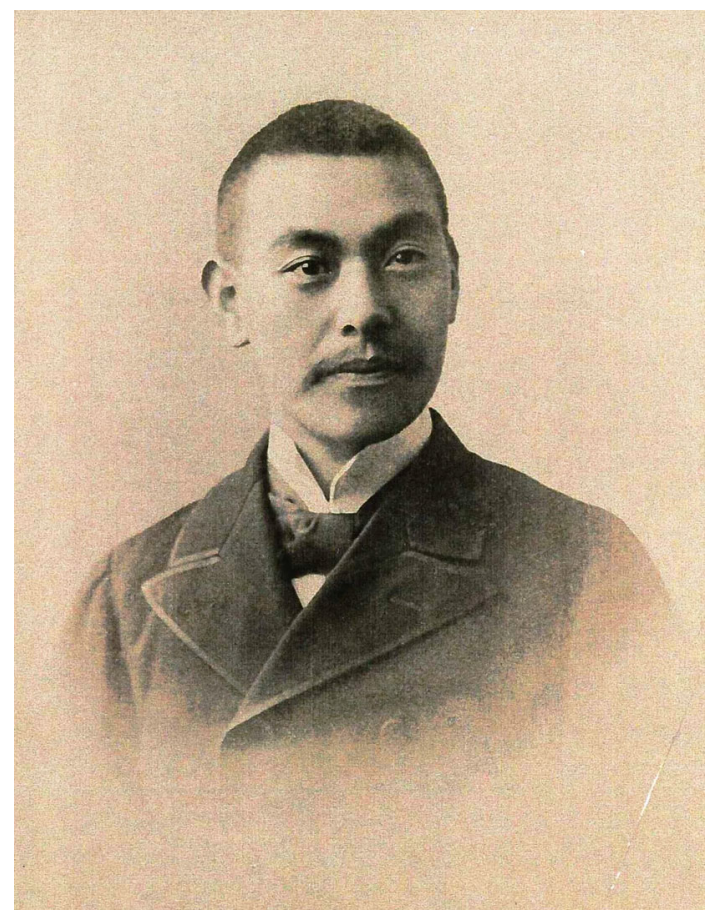

Abb. 3 Kitashimas Eintragung in das private Gästebuch der Familie Behring am 16. Dezember 1899 (C) Behring-Archiv Marburg, Sign. EvB/L 266

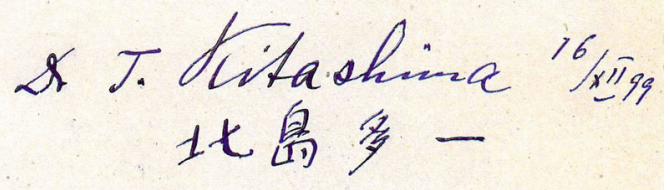

graphischen Lexika erwähnt (Hartmann 2017), wurde aber bisher, da keine Übertragung ins Deutsche vorlag, nicht rezipiert. In dem sechzehn kurze Kapitel umfassenden Büchlein kommt auch Kitashimas Zeit in Deutschland zur Sprache. Kitashima, der zur Zeit der Berichterstattung auf ein gesellschaftlich anerkanntes Leben zurückschaute - unter anderem war er seit 1931 Präsident der japanischen Ärzteverein (JMA) und wurde 1953 als „Person mit besonderen kulturellen Verdiensten“" [Bunka Kôrôsha] geehrt -, schildert mit der Erzählung dieses wichtigen Lebensabschnitts auch seine Wahrnehmung des damals schon weltberühmten Wissenschaftlers Behring, der bei ihm einen so nachhaltigen Eindruck hinterließ, dass er ihm in dem schmalen Erinnerungsband breiten Raum gewährt.

Neben den Erinnerungen enthält das Buch Kitashimas Lebenslauf und eine Liste seiner Publikationen. Trotz der zu beachtenden Einschränkung 
der zeitlichen Distanz von fünfzig Jahren - Kitashima war achtzig Jahre alt, als er seinen Schülern aus sein Leben berichtete - und der doppelten Vermittlung durch die von Schülern vorgenommene Verschriftlichung der mündlichen Erzählung gewähren die Erinnerungen einen Einblick in die Arbeits- und Forschungsbedingungen in Marburg, wie sie sich um 1900 darstellten.

Ishihara hat die Marburger Passagen ins Deutsche übertragen und dabei die besondere Ausdrucksweise der mündlichen Erzählung mit ihren stilistischen Besonderheiten wie Bekräftigung durch Wiederholung und Gedankensprünge beibehalten. Höflichkeit und Bescheidenheit, aber auch anhaltende Enttäuschung über Behring bedingen die sprachlichen Merkmale wie Andeutungen, Assoziationen und Auslassungen, die bei der Übertragung ins Deutsche zum besseren Verständnis des Gesagten ergänzt werden mussten.

Es ist zu bedenken, dass es sich bei dem vorliegenden Text um die Aufzeichnungen der Schüler handelt, die die mündliche Erzählung zu Papier brachten. Da Schüler in Japan traditionell in einem engen, ja ehrerbietigen Verhältnis zu ihren akademischen Lehrern standen und diese als Vaterfiguren ansahen, muss man davon ausgehen, dass die Niederschriften nicht frei von Färbungen sind, die auf diese patriarchalischen Prinzipien zurückzuführen sind (Chen 2010: 18; Donath 1998: 115; Miyajima 1935: 4).

\section{Übertragung}

Kitashima Taichi Jiden - Autobiographie von Taichi Kitashima Aus dem 4. Kapitel. Daigaku-Sotsugyôgo no Kenkyû: "Meine Forschungen nach dem Studienabschluss an der Tokio-Universität", Abschn. 2: DoitsuRyûgaku: "Der Aufenthalt in Marburg”

(S. 31-34: Volltext des Abschn. 2, Übertragung Aeka Ishihara)

[S. 31:] Inzwischen wurde es festgelegt, dass ich im Dezember ${ }^{8}$ Meiji 30 [= 1897] nach Deutschland fahren sollte, um bei Professor Behring Forschung zu betreiben.

Herr Behring war Ordinarius der Hygiene an der Universität Marburg. In der Direktion des Hygienischen Instituts wurde er aber vom außerordentlichen Professor Erich Wernicke vertreten, und Behring hielt dort nur selten Lehrveranstaltungen ab.

Stattdessen besaß Prof. Behring ein vortreffliches Privatlaboratorium auf dem Schlossberg. Dies war ein Geschenk der Farbwerke Höchst.

[S. 32:] Die Innenräume des Laboratoriums waren modern und sehr ordentlich eingerichtet. Die Geräte waren auf dem neuesten Stand. Nach 


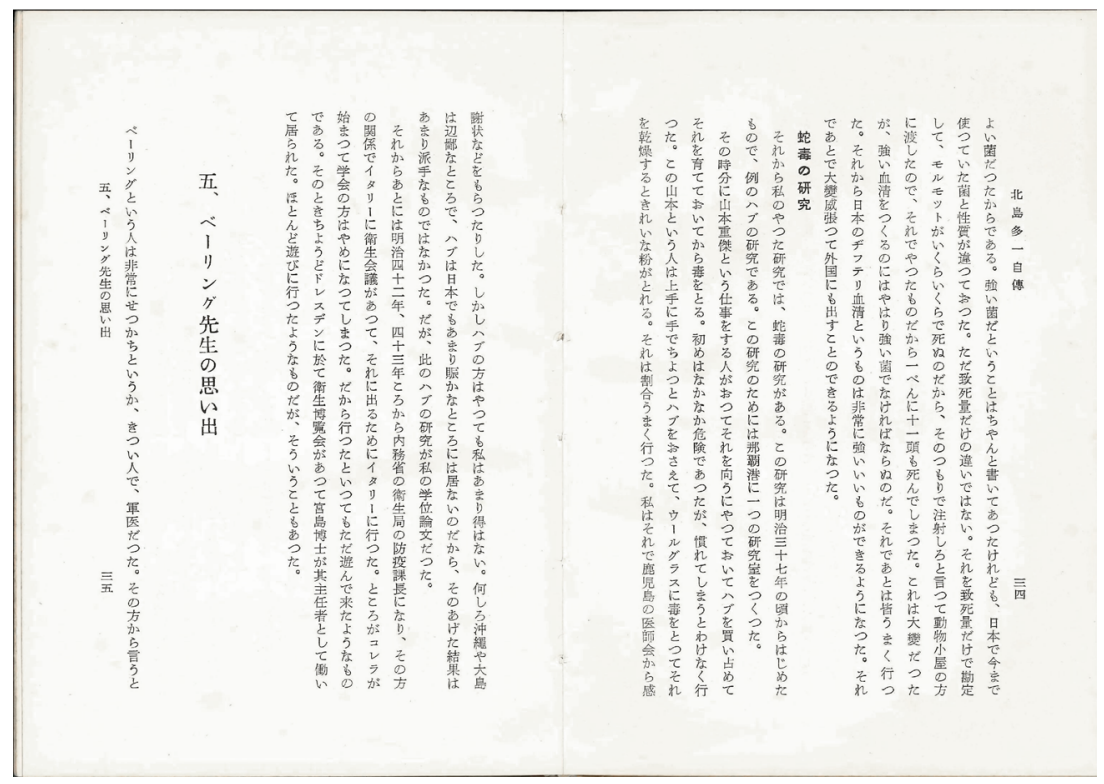

Abb. 4 Taichi Kitashima: Jiden, Doppelseite 34-35; Bildrechte () Kitasato-Institut; Abdruck mit freundlicher Genehmigung des Kitasato-Instituts Tokyo/Japan

meiner Ankunft in Marburg zeigte Behring zunächst kaum Interesse für mich, bat mich aber später, in diesem Schlosslaboratorium zu arbeiten. Dort arbeitete ein Chemiker namens Ruppel und beschäftigte sich mit der Bekämpfung der Tuberkulose, vor allem mit der Tuberkulin-Säure usw. Auf dem Gelände befand sich ein großes Gebäude mit einem ehemaligen Tanzsaal, wo man noch richtig tanzen konnte. Während die Bediensteten im zweiten Stock wohnten, stand mir die größere Wohnung im ersten Stock zur Verfügung. Ich zog schließlich dort ein, weil es einfach und für die Durchführung der Experimente sehr praktisch und bequem war. Behrings damaliger Forschungsschwerpunkt war die Tuberkulose, und ich habe auch mit Kühen, Gänsen und allen möglichen anderen Tieren experimentiert, aber leider hatte ich keinen guten Erfolg. Ich konnte nur kleine Nebenergebnisse vorweisen wie z. B. bei der Nukleinsäure.

[S. 33:] Es war einfach schade, dass ich in Deutschland erfolglos blieb. Die drei Jahre in Marburg waren für mich faktisch umsonst, ich habe nur Behring geholfen und für ihn gearbeitet. Mein Plan war gescheitert. Ich hoffte, selbst das Blutserum gegen die Tuberkulose zu entdecken und bemühte mich jeden Tag fleißig weiter, aber umsonst. Nach meiner Rückkehr nach Japan war ich immer noch damit beschäftigt, aber leider ohne Erfolg. Allerdings kümmerte ich mich in diesem Zeitraum um die Entwicklung des Nährbodens meines Kollegen Endô: ${ }^{9}$ Selbstverständlich war Herr Endô da- 
für zuständig, aber ich habe ihm ziemlich viel geholfen. Denn dieser Nährboden (bzw. dieses Substrat) ging auf eine Forschungserlaubnis von Yôjyôen [ein von Kitasato geleitetes privates Sanatorium, AI] zurück, hierbei war ich als Chef verantwortlich. Mein zweites dortiges Arbeitsziel war es, selbst Antitoxin mit guter Qualität zu gewinnen. Ich war daher selbst als Ingenieur und staatlicher Beauftragter im Kessei-Yakuin [Labor zur Herstellung von Blutserum, AI] tätig. Aus Marburg nahm ich das Diphtheriegift mit nach Japan und behandelte elf Pferde zum Zweck der Antitoxingewinnung. Vermutlich war das deutsche Gift zu stark, denn alle behandelten Pferde starben beim ersten Versuch. Diese Ergebnisse erschreckten nicht nur mich, sondern auch meinen Lehrer Kitasato. Elf Pferde sind gleichzeitig ums Leben gekommen. Das deutsche Gift hatte deutlich bessere Qualität als unser japanisches.

[S. 34:] Zwar stand auf dem Begleitzettel, dass das Gift stark und sehr wirksam sei, aber seine Eigenschaft unterschied sich vom japanischen Gift. Es geht nicht nur um die tödliche Dosis: Ich sagte: „Eine geringe Menge dieses Giftes ist für viele Versuchskaninchen tödlich." Ich habe der Aufzuchtfarm das Gift aus Deutschland gegeben, man hat bloß an die Dosis gedacht und nicht an die Wirkung, so dass man auf einmal elf Pferde verlieren musste. Man lernte aus den Fehlern und produzierte bald darauf ein sehr wirksames Antitoxin in Tokio. Wir sind darauf stolz und exportieren auch ins Ausland. [...].

Aus dem fünften Kapitel. Bêringu-sensei no Omoide: „Erinnerung an den Professor Behring"

(Ende S. 35 bis Anfang S. 39, Übertragung Ishihara)

[S. 35:] Behring war extrem ungeduldig und streng und Militärarzt von Beruf.

[S. 36:] Der Grund, weshalb die königliche Familie von Preußen den preußischen Stabsarzt Behring seinem Lehrer Robert Koch deutlich vorzog, war, dass Kochs zweite Frau von Beruf Tänzerin war. Wegen der unpassenden Herkunft seiner Frau wurde Koch zu den Veranstaltungen mit der königlichen Familie manchmal nicht eingeladen. Das tat mir sehr leid. Behring dagegen ging bei Hofe ein und aus.

Die Lehr- und Arbeitsmethode Behrings war folgende: Jeden Tag kam er zu uns und gab uns den Zettel, worauf stand, was wir heute machen sollten. Falls wir an diesem Tag nicht alles erledigen konnten, bekam er gleich schlechte Laune. Daher arbeiteten wir präzise, ziel- und zeitbewusst. $^{10}$ Die Arbeitstage verliefen immer so, vor allem für die deutschen Kollegen. Es gab eigentlich kaum einen Japaner, der so wie ich begünstigt wurde. Behring hat mich vor den anderen deutlich bevorzugt. Er ließ mich im Schlossberglaboratorium wohnen. Aus dieser Wohnung, in der 
ich einst gewohnt hatte, musste ich allerdings wegen der Schwangerschaft der Bediensteten - sie sagte, sie könne sich leider nicht mehr um mich kümmern - ausziehen und in der Stadt wohnen. Jeden Morgen hatte ich [im Schlossberglaboratorium] frischen Kaffee bekommen. In der großen Wohnung waren ein Kamin, ein großes Bett, ein großer Schreibtisch und Bücherregale vorhanden. Es war eine ausgesprochen schöne Wohnung.

[S. 37:] Wie gesagt, musste ich diese Wohnung wegen zwingender Umstände verlassen, es war schade. Behring war Militärarzt von Beruf, sehr streng und eine schwierige Person. Er hatte eine Vorliebe für die Mensur, und war darin so geschickt, dass seine Gegner einen schweren Stand gegen ihn hatten. ${ }^{11}$ Ich verhielt mich daher von Anfang an vorsichtig distanziert. Wahrscheinlich fand Behring an dieser Haltung Gefallen und hielt mich geeignet für die Mitarbeit an der Spitzenforschung. Gegenüber Nicht-Deutschsprachigen schien zudem das Risiko, wissenschaftliche Geheiminformationen durchsickern zu lassen, geringer. Zwar durfte ich mit den wenigen für Behring nützlichen fleißigen Mitarbeitern in den speziellen Räumlichkeiten arbeiten, zu denen sonst das Betreten streng verboten war, aber das bedeutet, dass ich total abgekapselt tätig sein musste, es war nämlich eine Art vom „Arrest“. Zunächst fand ich es nicht schlimm, so isoliert $\mathrm{zu}$ arbeiten (denn innerlich hatte ich mich zunächst auf eine enge wissenschaftliche Zusammenarbeit mit Behring gefreut), aber eigentlich habe ich zum Schluss Pech gehabt. Sehr viel Zeit habe ich in Deutschland umsonst vergeudet; ich habe sogar noch eine Weile nach meiner Rückkehr nach Japan hartnäckig versucht, im Yôjyô-en meine Forschung fortzusetzen, mit der (wie es sich zeigen sollte falschen) Hoffnung, bald in diesem Forschungsbereich eine wichtige Entdeckung zu machen. Nein, ich war dumm, es war mein Fehler! Obwohl es mich schon so viel Zeit gekostet hatte, habe ich gar nichts Wichtiges finden können, ich hätte darauf möglichst schnell verzichten müssen. Nicht nur ich, sondern auch Herr Ruppel $^{12}$ sowie einige Deutsche, die später kamen, wurden von Behring - genauso wie ich - ausgenutzt und opferten ihre kostbare Zeit für die Tuberkuloseforschung Behrings. Ruppel war eigentlich ein begabter Chemiker, hat aber gar nichts Großes tun können.

Behrings Privatlaboratorium befand sich in der Nähe vom Schloss, also auf dem Schlossberg.

[S. 38:] Die Stadtbewohner nannten es das „Pulverinstitut“, weil das mechanische Geräusch draußen wie das einer Mühle klang. Eigentlich züchtete man dort Tuberkelbazillen und sammelte dann deren Zellkörper, die man schließlich chemisch bearbeitete. Zwei Jahre lang arbeitete ich in diesem Laboratorium. In Japan habe ich weiter experimentiert, weil ich hoffte, doch noch etwas Nützliches daraus zu machen. Ich habe mit allen mögli- 
chen Tieren, auch Wassertieren [Gänsen, UE] und Vögeln, experimentiert, aber ohne Erfolg.

Als ich in Behrings Institut tätig war, war ich der einzige Japaner. Behring wollte keine Ausländer außer mir hereinlassen, selbst deutschen Wissenschaftler wollte er ungern Eintritt ins Institut gewähren. Die Arbeit im Laboratorium war völlig anders als die an der Universität. Am Anfang gingen wir auch an die Universität, aber bald befahl Behring uns, zu ihm zu kommen, so dass ich hauptsächlich in seinem Privatinstitut arbeitete. Zwar war ich an der Universität registriert, ging aber selten an die Universität, nur bei besonderen Gelegenheiten wie z. B. bei Vorlesungen und Vorträgen. Sonst arbeitete ich stets im Laboratorium. Damals dachte ich oft und denke es jetzt auch, dass das deutsche Volk ein besonderes Talent für Forschung hat. Deutsche sind meines Erachtens anders als Franzosen, anscheinend ist das deutsche Gehirn zur Forschung besonders geeignet. Während die Kunst und Musik in Frankreich besser entwickelt sind, ist das deutsche Volk unter den europäischen Völkern für die Forschung besonders begabt. Die deutschen Forscher haben durchschnittlich ein höheres Niveau.

[S. 39:] Daher glaubten die japanischen Offiziere, die einmal in Deutschland studiert hatten, dass Deutschland den zweiten Weltkrieg bestimmt gewinnen könnte. Dieser Gedanke beeinflusste Japan: Man glaubte irgendwie fest daran, dass Deutschland zwar den ersten Weltkrieg verloren hat, aber bestimmt den zweiten Weltkrieg gewinnen würde.

[Es folgt eine Aufzählung der Reisestationen in Europa während den Ferien, z. B. Paris, England, Spanien, Budapest; Kitashima unternahm die Reisen hauptsächlich in Bezug auf seine Tuberkulose-Forschung, AI.]

[S. 40:] Um am internationalen Tollwut-Symposium im Pasteur-Institut in Paris teilzunehmen, fuhr ich noch einmal nach Europa, und zwar mit Herrn Kusama, ${ }^{13}$ der im Anschluss daran in Amerika bleiben und dort seine Forschung treiben sollte. Daher habe ich mich von ihm in San Francisco verabschiedet. [...].

\section{Kommentar ${ }^{14}$}

\section{Das Schlossberglaboratorium}

Das im Text ausführlich geschilderte Schlossberglaboratorium war 1895/96 für Behring als Wohnhaus und privates Labor gebaut worden (Enke 2017), im angegliederten Maschinenhaus war eine als Blutschleuder dienende Zentrifuge untergebracht (Behring 1906: 333), die das von Kitashima erwähnte mechanische Geräusch erzeugte. Das komplexe, aus Gerätschaften, 
Tieren und Menschen bestehende Labornetzwerk ermöglichte in Kombination mit Tierställen und Wiesen für serumliefernde Großsäuger eine Arbeitsweise, die auf engstem Raum zu verwertbarem und auch kommerziell erfolgreichen anwendungsbezogenen Ergebnissen führen sollte. Finanziell wurde die Unterhaltung der von der Universität unabhängigen Forschungseinrichtung von den in Höchst am Main ansässigen Farbwerken vormals Meister Lucius \& Brüning unterstützt, die mit der Förderung die Hoffnung auf Entwicklung eines gewinnbringenden Heilmittels zur Behandlung der Tuberkulose verband.

\section{Das Arbeitsklima}

Die auf ökonomischem Ertrag ausgerichtete Zielsetzung spiegelt sich in Behrings auf Effizienz ausgerichteter Arbeitsweise wider. Von seinen Untergebenen erwartete er, wie auch die Äußerungen anderer Mitarbeiter zeigen, ${ }^{15}$ Unterordnung unter die Hierarchie und uneingeschränkten Dienst an der Sache. Das distanzierte und keineswegs teamorientierte Verhalten gegenüber Kitashima im wissenschaftlichen Umfeld war kennzeichnend für Behring. Dass es in anderen deutsch-japanischen Lehrer-Schüler-Beziehungen unterstützend und "freundlich“ zuging, zeigen Beispiele bei Chen (Chen 2010: 110 f.). Chen berichtet allerdings auch von schwierigen LehrerSchüler-Konstellationen, die erst mit dem Abstand mehrerer Jahre durch deutlich negative Zuschreibungen charakterisiert wurden (ebd.: 132). Behrings Haltung präsentiert sowohl sein am Ertrag orientiertes Arbeiten als auch seinen Führungsstil, der, wie auch andere ehemalige Assistenten in ihren Lebenserinnerungen berichten (Much 1932: 17), vom Einhalten einer festen Rangordnung geprägt war und wenig Platz für Eigeninitiative und individuell gewählte Forschungsschwerpunkte ließ (Schulze-Rath 1993: 108). Für den an einer allgemeinen Weiterbildung im Feld der Infektionskrankheiten und der Immunologie interessierten, zudem an die japanische Höflichkeit und Rücksichtnahme gewohnten Kitashima mag Behrings von starken Eigeninteressen gelenktes Regiment ein Affront gewesen sein, über den auch die privaten Einladungen in die Villa Behring nicht hinweg halfen. ${ }^{16}$ Im Rückblick mag Kitashima auch die Erfahrungen der Kitasato-Schüler Kiyoshi Shiga (1871-1951) und insbesondere Sahachiro Hata (1873-1938) zum Vergleich herangezogen haben. Die beiden japanischen Kollegen, die ab 1901 bzw. 1909 bei Paul Ehrlich in Frankfurt im Institutfür experimentelle Therapie arbeiteten, kamen mit vorzeigbaren Forschungsergebnissen aus Deutschland zurück und konnten auch auf ein menschlich angenehmes Klima im Umfeld Ehrlichs zurückblicken. So äußerte sich Ehrlich in Briefen an Dritte freundlich und dankbar über Hata, der von Ehrlich als Unterstützer im Forschungsalltag „wie ein Geschenk des Himmels“ (Hüntelmann 2011: 182) wahrgenommen wurde. 
Die Marburger Forschungsschwerpunkte

Nach seinen Erfolgen mit dem inzwischen industriell hergestellten Diphtherieantitoxin wandte sich Behring verstärkt der Rindertuberkulose- und Tuberkuloseforschung zu, die auch von den Farbwerken gefördert wurde. Darüber hinaus beschäftigten sich die Mitarbeiter des Privatlabors auch mit den Erregern von Cholera und (Pferde-)Rotz. Aus diesen Forschungen erwuchsen drei Publikationen, an denen Kitashima entweder als Mitautor oder als im Text namentlich erwähnter Mitarbeiter beteiligt war (Ransom \& Kitashima 1898; Behring 1899; Behring \& Kitashima 1901). Sowohl diese Publikationspraxis als auch ein Schreiben an Elias Metschnikoff (1845-1916), in dem Behring darum bittet, Kitashima im PasteurInstitut Prüfungen an Tetanusgift vornehmen zu lassen, ${ }^{17}$ zeigen, dass der als schroff und eigennützig bekannte Behring Kitashima als Wissenschaftler schätzte.

\section{Austausch und Vernetzung}

Betrachtet man den Deutschlandaufenthalt Kitashimas aus der globalen Perspektive, wird ein Netz sichtbar, das über Berlin, Tokio, Marburg und Paris gespannt ist. Zwischen den Knotenpunkten bewegen sich Akteure, die mit Hilfe eines "Schülers“ und dem Austausch von Gaben alte Kontakte auffrischen: Der „fleissigste Schüler“ - bereits mit der zum Ausdruck gebrachten Vorzüglichkeit des Abgesandten vermittelt sich eine Geste der Wertschätzung - wird „mit einem kleinen Geschenk von Japanischen Waaren" nach Marburg gesandt (Enke 2010: 178) und reist am Ende seines Europaaufenthaltes mit hochwirksamem Diphtheriegift im Gepäck nach Japan zurück. Den Einzugsbereich von Deutschland aus erweiternd wird dieser Schüler von einem anderen Akteur mit einer Empfehlung und Bitte nach Paris an das Institut Pasteur geschickt, womit gleichermaßen dem japanischen Lehrer als auch den Pariser Kollegen Respekt und Hochachtung gezollt werden. Der Schüler wiederum kann diese Kontakte nutzen, die einerseits sein Renommee in der Heimat steigern und andererseits weiteren weltumspannenden Begegnungen bis hin nach "San Francisco“ die Türen öffnen (Kitashima 1955: 40). Das anhand von Kontakten und durch Einblick in die „Privatlabore“ erlangte Wissen kann schließlich in der Heimat nutzbringend angewandt werden, was sich wiederum positiv auf die akademische Karriere auswirken wird.

Die nun nach Japan mitgegebene „Gabe“ (Mauss 1990 [1925]), die nicht nur als Geschenk und großzügige Geste an den Überbringer, sondern auch an den Initiator, nämlich Kitasato, verstanden werden kann, behält ihren Wert, auch wenn sie zunächst zum Verlust von elf Pferden führt. Mit dem Diphtherietoxin wird nicht nur ein Stoff, sondern auch ein implizites Wissen überreicht, das modifiziert gewinnbringend umgesetzt werden kann: 
„Man lernte aus den Fehlern und produzierte bald darauf ein sehr wirksames Antitoxin in Tokio“ (Kitashima 1955: 34).

\section{Vergeudete Zeit? - Ein Fazit}

Die Klage über die vergeudete Zeit in Marburg gehört zu den rätselhaftesten Textpassagen Kitashimas. Man kann nicht ausschließen, dass der Topos von Pech und Erfolglosigkeit der japanischen Höflichkeit und Bescheidenheit geschuldet ist, da sich ähnliche Äußerungen auch bei Sunao Tawara (1873-1952) in seinen Briefen an Ludwig Aschoff (1866-1942) finden. ${ }^{18}$ Aber wahrscheinlich hatten sowohl Kitashima als auch sein Mentor Kitasato erwartet, dass die erhoffte enge Zusammenarbeit mit dem Marburger Hochschullehrer, eventuell in Analogie zu den Berliner Forschungen des damals sehr erfolgreichen Teams Kitasato und Behring, zeitnah einen Durchbruch hinsichtlich Kitashimas eigenen Serumforschungen bringen würde.

Wie deutlich wurde, kann der Aufenthalt zunächst als Austausch von Gaben und damit als Verstetigung der Wissenschaftlerbeziehung zwischen Behring und Kitasato angesehen werden. Doch auch bezogen auf Kitashima selbst, der hier zunächst als Spielball erscheint, lässt sich in Hinblick auf den weiteren Verlauf seiner beruflichen Karriere das Diktum von der vergeudeten Zeit in Frage stellen. Käser hat darauf hingewiesen, dass in der Meiji-Zeit nur diejenigen Japaner Hochschullehrer wurden, die bei deutschen Medizinern in Japan oder Deutschland studiert hatten. Ein mit einer wissenschaftlichen Weiterbildung verbundener Aufenthalt in Europa war somit eine unabdingbare Voraussetzung für eine akademische Laufbahn. Die Auslandsstudenten wurden nach ihrer Rückkehr aus Europa zunächst Assistenten oder Dozenten an der Medizinischen Fakultät in Tokio, einige wurden schließlich auch Professoren (Käser 2011: 116; Chen 2010: 16). Die Japaner hatten nicht nur ein Wissensmonopol, sondern profitierten auch vom guten Ruf ihrer Gastgeber (Hüntelmann 2011: 308). Kitashimas eigene berufliche Entwicklung bestätigt diese Beobachtung.

Wenige Wochen, nachdem Kitashima im April 1901 wieder in Japan angekommen war, wurde er am 14. Mai 1901 Abteilungsvorsteher des Kaiserlichen Instituts für Infektionskrankheiten und Leiter der staatlichen Serumproduktionsstätte (Kessei-Yakuin) sowie außerordentlicher Quarantäne-Offizier. Ab September 1917 bekleidete er eine Professur an der Medizinischen Fakultät der (privaten) Keiô-Universität. Von Mai 1928 bis 1944 war er Dekan der dortigen Medizinischen Fakultät. Anschließend wurde er zunächst Vizedirektor und 1931 Direktor des (privaten) Kitasato-Instituts 
(bzw. des Kitasato-Forschungsinstituts) in Tokio. Im gleichen Jahr wurde er zum Präsidenten der japanischen Ärztevereinigung (Japan Medical Association: JMA) gewählt. 1953 verlieh man ihm die eingangs erwähnte Auszeichnung als Person mit besonderen kulturellen Verdiensten. ${ }^{19}$

Auch die bei der Entwicklung eines Schlangengiftserums praktisch angewandten serumtherapeutischen Fertigkeiten verdeutlichen, dass das in Europa erworbene Laborwissen in Japan mit Erfolg umgesetzt wurde: Vermutlich angeregt durch den Austausch mit dem Bakteriologen Albert Calmette (1863-1933), ${ }^{20}$ den er beim Besuch des Pariser Institut Pasteur kennen gelernt hatte, untersuchte Kitashima 1902 das Gift der Habu (Trimeresurus flavoviridis), einer auf der japanischen Insel Amami heimischen hochgiftigen Schlangenart. Es gelang ihm, ein äußerst wirksames Antiserum gegen das schnell und intensiv wirkende Gift $\mathrm{zu}$ entwickeln, das viele Menschenleben rettete. ${ }^{21}$ Für seine in japanischer Sprache verfasste Abhandlung Habudoku no Kessei oyobi sono Kessei-Ryôhô ni tsuite [deutsch: Studien über das Habu-Gift und die Behandlung durch Antitoxin] (Kitajima 1908) erhielt er 1909 den Asakawa-Forschungspreis.

In den folgenden Jahren hielt Kitashima auch über Behrings Tod hinaus Kontakt nach Marburg. Fotos und Eintragungen in das private Gästebuch der Familie dokumentieren Besuche in den Jahren 1911 und 1927. 1932 schickte er auf Wunsch Else von Behrings seine für eine Behring-Biographie bestimmten Erinnerungen nach Deutschland (Enke 2014: 226). Das in den 1940er Jahren in Japan aufflammende politisch motivierte Bekenntnis zu den deutsch-japanischen Wissenschaftsbeziehungen fand auch seinen Ausdruck in öffentlichen Erinnerungsfeiern für Behring, denen der deutsche Botschafter Eugen Ott (1889-1977) beiwohnte. Der fünfzigste Jahrestag der Entdeckung der Serumtherapie bot dem nationalsozialistischen Staat 1940 die Gelegenheit, die historischen Verbindungen nun unter dem ideologischen Schirm des Dreimächtepakts Berlin, Rom und Tokio zu reaktivieren. Ausführungen in der von Alexander von Engelhardt (1885-1960) 1942 herausgegebenen Jubiläumsschrift Die Welt dankt Behring zufolge erwähnte Kitashima bei seiner Ansprache anlässlich der am 16. Oktober 1940 in Tokio stattfindenden Gedächtnisfeier nicht nur die wissenschaftlichen Leistungen von Behring und Kitasato, sondern explizit auch das gerade zustande gekommene ,japanisch-deutsch-italienische Bündnis“ (Engelhard 1942: 64 f.). Die Kontinuität sowohl der vergangenen als auch der wiederbelebten deutsch-japanischen Kooperation drückte sich in der Anwesenheit der Gäste Fritz von Behring und Shutarô Kitasato aus, den jeweils ältesten Söhnen von Behring und Kitasato. Nach dem Zweiten Weltkrieg nahmen Deutschland und Japan erst 1955 wieder diplomatische Beziehungen auf, dem Jahr, in dem Kitashima seine Erinnerungen zu Papier brachte (Abb. 5). 


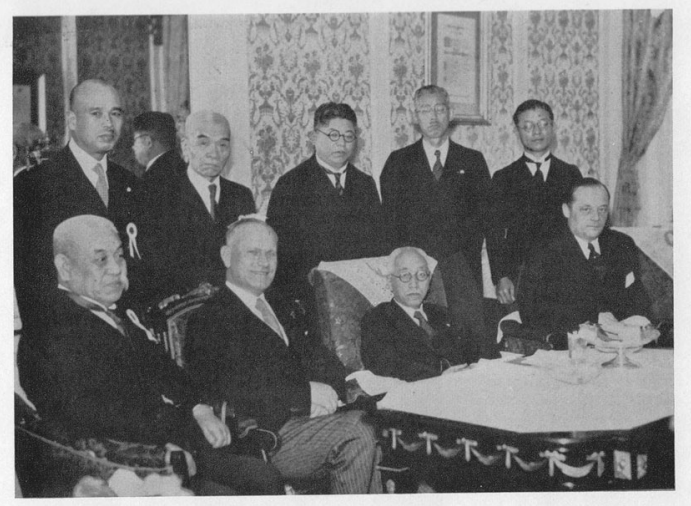

GE D A CHTN IS F E I E R ZUM 50. JAHRESTAG DER ENTDECKUNG DER SERUM-HEILKUNDE Von links nach rechts sitzend: Dr. Taichi Kitashima, der gegenwärrige Leiter des Kitazato Versuchslaboratorums und Dekan der medizinischen Fakultat an der Keio Universitat, der deutsche Botschnfter Eugen Ott, Erziehungsminister Kunihiko Hashida, Dr. Fritz von Beh-
ring, Sohn des verstorbenen Dr. Emil Adolph Behring. ring, Sohn des verstorbenen Dr. Emil Adolph Behring.
Stehend: Dr. Yoneji Mijakawa, Direktor des LaboratoStehend: Dr. Yoneji Mijakawa, Direktor des Laborato-
riums für Infektionskrankheiten, Dr. Eigoro Kanasugi, Präsident der medizinischen Universität Jochi, Mr. Shuntaro Kitazato, ältester Sohn des verstorbenen Dr. Kitazato, Wohlfahrtsvizeminister Massuke Kodama und Dr. Zenjiro Kitazato, ein weiterer Sohn des verstorbenen Forschers, der den lieruf seines Vaters ergriffen hat.

Abb. 5 Ausschnitt aus „Die Gedächtnisfeier in Japan am 16. Oktober 1940", in: Alexander von Engelhard (Hg.) (1942). Die Welt dankt Behring (S. 64), Taichi Kitashima sitzend links im Bild, Fritz von Behring ganz rechts am Tisch

\section{Danksagung}

Die Autorinnen danken der Kitasato-Shibasaburô-Gedenkstätte in Tokio und dem Institute of Medical Science der Universität Tokio für die freundlich gewährte Akteneinsicht. Emilio von Behring, der Enkel Emil von Behrings, hat uns private Briefe seiner Großmutter Else von Behring zur Verfügung gestellt, wofür wir herzlich danken. Den beiden anonymen Gutachtern sind wir für die konstruktiven Anmerkungen und hilfreichen Rückfragen dankbar. Die Entstehung dieses Textes betrachten wir als einen gelebten Beitrag zum aktuellen deutsch-japanischen Wissensaustausch.

\section{Anmerkungen}

1 Hubensdorf (2006); historisch: Miyajima (1935). - Gemäß europäischer Gepflogenheiten werden zuerst die Vornamen und dann die Nachnamen genannt. Die Umschreibung folgt den im Marburger Behring-Nachlass verwahrten Quellen; hier Kitashima statt Kitajima, Kitasato statt Kitazato.

2 Nach japanischen Quellen: [Zeitschrift für Bakteriologie in Japan] 1897. - Die Abreise nach Japan muss im März erfolgt sein. Kitashima kam am 16. April 1901 im Hafen von Kobe an und setzte von dort aus seine Reise mit dem Zug in Richtung Tokio fort (Saikingaku-Zasshi 1901).

3 Zum gesellschaftlichen Leben und Alltag in dem bei den Japanern der dritten Gruppe beliebten Berlin siehe Chen (2010: 133-138). Chen spricht von „Studentenkolonie“ (ebd.: 140).

4 Über ihren wissenschaftlichen Werdegang informieren die den Dissertationen beigefügten Lebensläufe (Osaki [Ozaki] 1902; Tsuzuki 1900; Watanabe 1902). (Sonderdrucksammlung der Emil-von-Behring-Bibliothek, Marburg.) 
$5 \quad$ Else von Behring an ihre Mutter Elise Spinola, 21.11.1897. Nachlass Else von Behring (Privatbesitz Emilio und Tomas von Behring).

6 Ransom \& Kitashima (1898); Behring (1899); Behring \& Kitashima (1901).

7 Person mit besonderen kulturellen Verdiensten 2017. URL: https://de.wikipedia.org/ wiki/Person_mit_besonderen_kulturellen_Verdiensten (28.01.2017).

8 Kitashima reiste bereits im Oktober 1897 aus Japan ab.

9 Shigeru Endô (1870-1937) war seit 1894 im Institut für Infektionskrankheiten tätig. 1903 entdeckte er 1903 das später nach ihm benannte Endo-Agar (Lactose-FuchsinSulfit-Agar), mit dessen Hilfe man unter anderem Typhus erkennen und unterscheiden kann.

10 Diese Arbeitsabläufe beschreibt Kitashima auch in den Erinnerungen, die er 1932 an Else von Behring schickte (Zeiss \& Bieling 1941: 540). Zur Überlieferung Enke (2014).

11 In Berlin war Behring Mitglied, später Ehrenmitglied des Pepinière-Corps Suevo-Borussia. Bisher gibt es keine Quellen, die ihn als praktizierenden Fechtkämpfer mit scharfen Waffen (Mensur) beschreiben, vielleicht gab es jedoch Ereignisse in Berlin, die Kitasato bekannt waren und von denen er Kitashima berichtet hatte. In seinen sozialen und geschäftlichen Kontakten blieb Behring der von der Studentenverbindung geforderten Haltung (Contenance), also Beherrschung von Affekten, Standhaftigkeit und Nicht-Zurückweichen trotz drohender Verletzung, sein Leben lang treu.

12 Der aus Breslau stammende Chemiker Wilhelm G. Ruppel (1865-1925) hatte in Freiburg im Breisgau studiert und dort 1889 seine Dissertation Ueber das Di- $\beta$-naphtylenketonoxyd vorgelegt. Seit dem Sommersemester 1900 arbeitete Ruppel als Abteilungsvorsteher am Hygienischen Institut der Universität Marburg; 1902 erhielt er den Titel „Professor". Seine wissenschaftlichen Leistungen lagen auf dem Gebiet der Serumtherapie und der Tuberkulosebekämpfung. Nach seiner Tätigkeit an der Universität Marburg wechselte er in die Industrie, lange Zeit leitete er die Serobakteriologische Abteilung der Farbwerke in Höchst. Seit 1918 stand er der Serobakteriologischen Abteilung der Elektroosmose-Aktiengesellschaft in Berlin vor. (Zusammengestellt aus Dokumenten des Behring-Archivs Marburg.)

13 Yoshio Kusama (1888-1968), japanischer Mediziner. Der erste Studienaufenthalt Kusamas in den USA erstreckte sich von 1916 bis 1920 mit Abschluss an den Universitäten Stanford sowie Johns Hopkins. 1926 folgte ein weiterer Forschungsaufenthalt in den USA. Kusama wurde Dekan der Medizinischen Fakultät der Keiô-Universität. Nach dem Zweiten Weltkrieg führte er ein amerikanisches Medizin-Ausbildungssystem in Japan ein. - 1927 besuchte Kusama Marburg und trug sich mit einer Widmung in das Gästebuch der Familie Behring ein (Behring-Archiv Marburg, Sign. EvB/L 266).

14 Der Kommentar wurde von den Autorinnen anhand japanischer und deutscher Quellen gemeinsam erarbeitet und von Ulrike Enke zusammengestellt.

15 Vgl. beispielsweise die Äußerungen Wilhelm Ruppels gegenüber Arnold Libbertz vom 16. September 1902, Behring erwarte, dass die Angestellten ihm „subordiniert" seien. Behring-Archiv Marburg, Sign. EvB/B 196/46.

16 Gästebuch der Familie Behring: 16. Dezember 1899, 12. Mai 1900. - Im Dezember 1899 trägt er sich als „Dr. T. Kitashima“ auf Deutsch und Japanisch ins Gästebuch ein. (Behring-Archiv Marburg, Sign. EvB/L 266)

17 Emil von Behring an Elias Metschnikoff, 28. Juli 1900. (Behring-Archiv Marburg, Sign. $\mathrm{EvB} / \mathrm{B} 1 / 99$.)

18 Tawara an Aschoff, 8. Oktober 1905: „[... ] wenn mir dann meine missglückten Versuche vor Augen standen, dann war ich tief unglücklich. Ich habe in den zwei langen Jahren keinen frohen Tag verlebt.“ Tawara an Aschoff, 12. Juli 1906: „Leider war meine wissenschaftliche Tüchtigkeit zu gering, mein Deutsch zu schwach [...]." (Suma, Shimada \& Shimada 2003: 210, 214). 
19 Kitashima [ohne Jahreszahl, „Akten Lebenslauf“]; „Kitasatos Schüler“ 2014. Diese Angaben unter Quellen. Die Quelleneinsicht erfolgte mit freundlicher Genehmigung der Kitasato-Shibasaburô-Gedenkstätte, Tokio, und dem Institute of Medical Science, Universität Tokio.

20 Calmette arbeitete seit 1890 mit Louis Pasteur (1822-1895) und Émile Roux (1853-1933) zusammen. Im Auftrag Pasteurs gründete er 1891 in Saigon eine Zweigstelle des Pasteur-Instituts und wählte als Hauptforschungsgebiet die Toxikologie. Zurückgekehrt nach Paris entwickelte er das "Calmette-Serum" gegen die Gifte von Echten Kobras (Elapidae, Giftnattern), siehe Calmette 1894.

21 Kitashima reiste im Mai 1905 mit seinem Serum auf die Insel Amami und nach Okinawa; dort konnte er es mit gutem Erfolg anwenden: Von 118 Gebissenen, die mit dem Serum behandelt wurden, konnten 113 geheilt werden (Kitashima 1908). Zur Schlangengiftforschung in Japan siehe Sawai (1966).

\section{Quellen}

Behring-Archiv Marburg: Nachlass Emil von Behring, www.uni-marburg.de/behring-digital Behring-Archiv Marburg: Sonderdrucksammlung

Magistrat der Stadt Marburg, Fachdienst Bauaufsicht, Bauzeichnungen des Gebäudes Gisonenweg 5 (ehemals Breiter Weg 5) [= Schlossberglaboratorium], Int.-Nr. 62a/5, 1896/97

Material für „Kitasatos Schüler. Sonderausstellung im Sagamihara Stadtmuseum in Kanagawa" vom 25. Oktober bis 16. November 2014 (im Besitz der Kitasato-ShibasaburôGedenkstätte, mit freundlicher Genehmigung)

Nachlass Else von Behring (Privatbesitz der Familie von Emilio und Tomas von Behring)

The Institute of Medical Science, The University of Tokyo: Kitashima, Taichi [1892-1914]: „Akten in der Zeit des Kaiserlichen Instituts für Infektionskrankheiten/Lebenslauf Kitashima" (mit freundlicher Genehmigung)

\section{Literatur}

Behring, Emil und Shibasaburô Kitasato 1890. Ueber das Zustandekommen der DiphtherieImmunität und der Tetanus-Immunität bei Thieren. Deutsche Medizinische Wochenschrift (49): 1113-1114.

Behring, Emil 1899. Ueber die quantitativen Bindungsverhältnisse zwischen Tetanusgift und Tetanusantitoxin im lebenden Meerschweinkörper. Von Prof. E. Behring, Marburg, nach Versuchen von Dr. Ransom und Dr. Kitashima. Fortschritte der Medicin (22): 521-534.

Behring, Emil von und Taichi Kitashima 1901. Ueber Verminderung und Steigerung der ererbten Giftempfindlichkeit. Berliner Klinische Wochenschrift (6): 157-163 [als Sonderdruck im Behring-Archiv Liste 04-002, Stand: A-I, Nr. 6/16, 16 S.].

Behring, Emil von 1906. Therapeutische Tierexperimente im Dienste der Seuchenbekämpfung. Der Mensch und die Erde (2): 331-354 und Beilage II.

Burks, Ardath W. (Hg.) 1985. The Modernizers: Overseas Students, Foreign Employees, and Meiji Japan. Boulder, London: Westview Press.

Calmette, Albert 1894. Propriétés du sérum des animaux immunisés contre le venin de serpents et thérapeutique de l'envenimation. Comptes rendus de l'Académie des sciences (118): 720-722.

Chen, Hsiu-Jane 2010. „Eine strenge Prüfung deutscher Art“. Der Alltag der japanischen Medizinausbildung im Zeitalter der Reform von 1868 bis 1914. Husum: Matthiesen. 
Donath, Diana 1998. Zur Kultur und Geschichte Japans. Ausgewählte Essays. Münster: LIT Verlag.

Engelhardt, Alexander von (Hg.) 1942. Die Welt dankt Behring. Berlin: Bruno Schultz.

Enke, Ulrike 2010. Schüler und Kollegen - Emil von Behrings Zusammenarbeit mit Shibasaburo Kitasato und Taichi Kitashima im Spiegel ihrer Briefe. In: Andreas Mettenleiter (Hg.). Japan - Siebold - Würzburg. 25 Jahre Siebold-Gesellschaft - 15 Jahre SieboldMuseum. Würzburg: Adamos: 175-187.

Enke, Ulrike 2014. Behrings Nachlässe - Behrings Biographien. Berichte zur Wissenschaftsgeschichte (37): 216-239.

Enke, Ulrike 2017. Zur Frühgeschichte von Behrings Institut für experimentelle Therapie, einem Wohnhaus und Forschungsinstitut auf dem Marburger Schlossberg. In: Philipps-Universität Marburg (Hg.). Baugeschichte der Philipps-Universität Marburg [zum Druck angenommen, erscheint 2017].

Hartmann, Rudolf 2000. Japanische Studenten an der Berliner Universität: 1870-1914. Berlin: Mori-Ôgai-Gedenkstätte der HUB. 2. überarb. u. erg. Aufl.

Hartmann, Rudolf, o. J. Japans Studierende in Deutschland 1868-1914. Ein Lexikon. URL: http://crossasia.org/digital/japans-studierende/index/show (31.01.2017).

Hubensdorf, Michael 2006. Kitasato Shibasaburō. In: Wolfgang U. Eckart und Christoph Gradmann (Hg.). Ärzte-Lexikon. Von der Antike bis zur Gegenwart. 3. Aufl. Berlin, Heidelberg: Springer: 191-192.

Hüntelmann, Axel C. 2011. Paul Ehrlich. Leben, Forschung, Ökonomien, Netzwerke. Göttingen: Wallstein.

Ishihara, Aeka 2012a. Japanische Medizinerinnen in Deutschland 1890-1905. Mizuko Takahashi und Tada Urata. Hiyoshi-Studien zur Germanistik (49): 75-101.

Ishihara, Aeka 2012b. Dokutorutachi no Huntôki. Goethe ga michibiku Nichidoku-IgakuKouryû. [Die immer strebenden Geister der Medizin. Der akademische Austausch zwischen Japan und Deutschland in der Meiji-Zeit]. Tokio: Keiô-University-Press [auf Japanisch].

Käser, Frank 2011. Medizin nach deutschem Muster. In: Curt-Engelhorn-Stiftung für die Reiss-Engelhorn-Museen und Verband der Deutsch-Japanischen Gesellschaften (Hg.). Ferne Gefährten. 150 Jahre deutsch-japanische Beziehungen. Begleitband zur Sonderausstellung. Mannheim: Schnell und Steiner: 113-117.

Kitashima, Taichi 1908. Habudoku no Kessei oyobi sono Kessei-Ryôhô ni tsuite [Studien über das Habu-Gift und die Behandlung durch Antitoxin.] Saikingaku-Zasshi 細菌學雑誌』[Zeitschrift für Bakteriologie in Japan] (154): 541-556 [auf Japanisch].

Kitashima, Taichi 1955. Kitashima-Taichi Jiden. Tokio: Kitashima Sensei Kinen Jigyôkai [auf Japanisch].

Kreuder-Sonnen, Katharina 2012. Wie die Mikroben nach Warschau kamen. Wissenstransfer in der Bakteriologie in den 1880er-Jahren. NTM - Zeitschrift für Geschichte der Wissenschaften, Technik und Medizin (20): 157-180.

Mauss, Marcel 1990 [1925]. Die Gabe. Form und Funktion des Austauschs in archaischen Gesellschaften. Frankfurt am Main: Suhrkamp.

Miyajima, Mikinosuke 1935. Lehrer und Schüler. Tokio [Selbstverlag].

Much, Hans 1932. Arzt und Mensch. Das Lebensbuch eines Forschers und Helfers. Dresden: Reissner.

Oberländer, Christian 1995. Zwischen Tradition und Moderne: Die Bewegung für den Fortbestand der Kanpô-Medizin in Japan. Stuttgart: Steiner.

Osaki [Ozaki], Sutezo 1902. Anatomische Untersuchung eines sympathisirenden Auges nebst Bemerkungen über das Zustandekommen der Chorioretinitis sympathica. Marburg. Diss. med. Universität Marburg.

Ransom, Frederick und Taichi Kitashima 1898. Untersuchungen über die Agglutinationsfähigkeit der Choleravibrionen durch Choleraserum. Deutsche Medicinische Wochenschrift (19): 295-296.

Reißenweber, Heidrun 2005. Japanische Medizin. In: Werner E. Gerabek, Bernhard D. Haage, Gundolf Keil und Wolfgang Wegner (Hg.). Enzyklopädie Medizingeschichte. Berlin: de Gruyter: 688-694.

Saikingaku-Zasshi 1897. 細菌學雑誌』Zeitschrift für Bakteriologie in Japan. 23: 51 und 26: 49. Saikingaku-Zasshi 1901. 細菌學雑誌』Zeitschrift für Bakteriologie in Japan. 65: 82. 
Sawai, Yoshio (Hg.) 1966. Bibliography of Snake Venom and Snakes in Japan and Taiwan. Laboratory of Biological Products Institute for Infectious Diseases. Tokio: The University of Tokyo. Nippon Nettai Igaku Kyôkai [Die Gesellschaft der Tropische Medizin in Japan] (auf Englisch und Japanisch).

Schulze-Rath, Renate 1993. Hans Much (1880-1932). Bakteriologe und Schriftsteller. Diss. med. Universität Mainz.

Suma, Kôzô, Munehiro Shimada und Tatsuo Shimada (Hg.) 2003. Sekai no shinzōgaku wo hiraita Tawara Sunao no shōgai. Tokio: Mikurosukopia shuppankai [mit einem Abdruck der auf Deutsch geschriebenen Briefe Tawaras an Ludwig Aschoff]

Tsuzuki, Jinnosuke 1900. Beitrag zur Tetanusantitoxintherapie bei Thieren und beim Menschen. Marburg: C. L. Pfeil.

Watanabe, Hiroshi 1902. Ueber eine schnellwachsende Struma in der Schwangerschaft mit tötlichem Ausgang. Leipzig: Georgi.

Zeiss, Heinz und Richard Bieling 1941. Behring. Gestalt und Werk. 2. Aufl. Berlin: Bruno Schultz.

Ulrike Enke

Emil-von-Behring-Bibliothek, Arbeitsstelle für Geschichte der Medizin

Philipps-Universität Marburg

Bahnhofstraße 7

35037 Marburg

Deutschland

ulrike.enke@staff.uni-marburg.de

Aeka Ishihara

Graduate School of Arts and Sciences (Komaba-Campus)

The University of Tokyo

Meguro-ku, Komaba 3-8-1

J153-8902 Tokyo

Japan 